\title{
¿Estamos como estamos porque somos como somos? Importancia de los indicadores socio-ambientales para un desarrollo a escala humana
}

\author{
Christian Henríquez. Zuñiga \\ Centro de Estudios Ambientales de la Universidad Austral de Chile (UACh) \\ Guillermo Pacheco Habert \\ Centro de Estudios Ambientales de la Universidad Austral de Chile (UACh)
}

Recebido: 28/01/2015 Versão revisada (entregue): 06/05/2015 Aprovado: 08/05/2015

\begin{abstract}
Resumen
Discutir sobre procesos de desarrollo y sustentabilidad posibilita reflexionar sobre los indicadores objetivos y subjetivos, como también revelar la importancia de la utilización de indicadores socio-ambientales, que permitan mejorar la comprensión de aquello que llamamos desarrollo y apellidamos de sustentable. El uso del PIB como indicador, para distinguir entre países desarrollados o en desarrollo, es problematizado y discutido en este trabajo. En esta dirección se presenta una discusión teórica que permite ampliar la mirada sobre los indicadores socio-ambientales con miras a un otro desarrollo. El artículo se nutre de investigaciones bibliográficas y empíricas realizadas en el contexto de una tesis de magíster que se amplían ahora desde en una tesis doctoral enmarcada en un proyecto Fondecyt. Se concluye que el PIB se ha utilizado por un largo periodo para medir la riqueza de las naciones, siendo objeto de críticas debido a su limitada cobertura de aplicación, que no responde a las necesidades contemporáneas. Los indicadores socio-ambientales de carácter participativo pueden mejorar la comprensión de los procesos de desarrollo territorial sustentable, desde una perspectiva crítica, transdisciplinaria y a escala humana.
\end{abstract}

Palabras-clave | Desarrollo a escala humana; desarrollo regional sustentable; indicadores socio-ambientales.

Código JEL | O15; Q01; Q56.

\section{ARE WE LIKE AS WE BEHAVE? IMPORTANCE OF SOCIO-ENVIRONMENTAL INDICATORS FOR HUMAN SCALE DEVELOPMENT}

\section{Abstract}

Discussing about development processes can generate a reflection about objective and subjective indicators, as well as revealing the importance of using socio-environmental 
indicators for understanding what we call as development. Using GDP as an indicator, or rather as a "marker" to differentiate between developed and developing countries, is discussed in this paper. It is present in this context a theoretical discussion which allows reflecting about socioenvironmental indicators, or on a different development. This article mix exploratory studies and bibliographic research, it has been made in the context of a master's dissertation. It concludes that the GDP has been used for a long time to measure the wealth of nations, and finally has been subject to criticism due to its limited global application, what does not answer contemporary needs. The socio-environmental indicators can improve the understanding of the processes for regional sustainable development, from a critical way, interdisciplinary and on a human scale.

Keywords | Human scale development; socio-environmental indicators; sustainable regional development.

JEL-Code | O15; Q01; Q56.

\section{ESTAMOS COMO ESTAMOS PORQUE SOMOS COMO SOMOS? IMPORTÂNCIA DOS INDICADORES SOCIOAMBIENTAIS PARA O DESENVOLVIMENTO EM ESCALA HUMANA}

\section{Resumo}

Discutir processos de desenvolvimento e sustentabilidade permite refletir sobre os indicadores objetivos e subjetivos, como também revelar a importância do uso de indicadores sociais e ambientais para melhorar a compreensão do que chamamos de desenvolvimento e apelidamos de sustentável. A utilização do PIB como indicador para distinguir entre países desenvolvidos e em desenvolvimento é problematizada e discutida neste trabalho. Neste sentido se apresenta uma discussão teórica que permite ampliar a perspectiva sobre os indicadores socioambientais, com vistas a um outro desenvolvimento. $\mathrm{O}$ documento se alimenta de pesquisa bibliográfica e empírica, iniciada no contexto de uma dissertação de mestrado e que agora se estende a um projeto de tese de doutorado, no âmbito de um projeto Fondecyt. Conclui-se que o PIB, usado por um longo período para medir a riqueza das nações, vem sendo objeto de crítica por causa de sua limitada cobertura de aplicação, que não responde às necessidades contemporâneas. Os indicadores de participação socioambientais podem melhorar a compreensão dos processos de desenvolvimento territorial sustentável, a partir de uma perspectiva crítica, transdisciplinar e em escala humana.

Palavras-chave | Desenvolvimento em escala humana; desenvolvimento regional sustentável; indicadores socioambientais.

Código JEL | O15; Q01; Q56.

\section{Introdução}

¿Qué entendemos por desarrollo?, o más bien, ¿Qué es desarrollo sustentable?, ¿Por qué tantas definiciones para estos conceptos? Si complejizamos un poco más y nos insertamos en las variables del territorio ¿Que sería desarrollo territorial sustentable? ¿Es posible medir ese desarrollo territorial sustentable?, Si es así 
¿Cómo medirlo?, este y otro interrogantes fueron las que motivaron las primeras reflexiones sobre la temática y el surgimiento de este artículo y de esta investigación (HENRÍQUEZ, 2009).

Vivimos inmersos dentro de un modelo de "desarrollo" económico hegemónico desigual, donde constantemente el desarrollo es sinónimo de crecimiento económico. Aquí se discute tal afirmación, porque creemos que el desarrollo tiene que ver con las personas y no con objetos, y los actuales indicadores macroeconómicos no se refieren a personas y menos a la naturaleza (MAXNEEF, 2001). Para Sen (2000) el desarrollo consiste en la eliminación de las privaciones de libertad que limitan las alternativas y oportunidades de las personas para ejercer responsablemente su condición de agentes.

El Siglo XX fue testigo de significativas transformaciones en todas las dimensiones de la existencia humana (HOBSBAWM, 1996; VAN BELLEN, 2006). Estas transformaciones son resultado de un modelo de desarrollo hegemónico, economicista y utilitarista de los países del hemisferio norte, denominados desarrollados (países del centro), que se imponen sobre los países del hemisferio sur, denominados subdesarrollados o en desarrollo (países periféricos).

Este modelo de desarrollo está inspirado y controlado muchas veces en un modelo maligno de inversión extranjera directa, que en vez de potenciar el desarrollo acaba por entorpecerlo (MORAN, 2000).

Según Rojas (2008), la construcción de políticas públicas se basa en demandas que aspiran a un mayor bienestar de las poblaciones. Para este propósito se elaboran y construyen indicadores objetivos, que se supone, se encuentran estrechamente relacionados con el bienestar de la población en territorios específicos. Estos indicadores permiten medir, por ejemplo, el éxito o fracaso de una determinada política pública.

Según Max Neef $(2001,2007)$, nos encontramos frente a un modelo hegemónico, que se basa en indicadores objetivos, de los que se destacan el nivel de ingreso, la tasa de crecimiento anual, la tenencia de bienes duraderos y el acceso a algunos servicios públicos. En este sentido, el Producto Interno Bruto (PIB) guía la toma de decisiones en la elaboración de la gran mayoría de las políticas públicas y planes de inversión de cada país.

Se trata, según Dupas (2005), de un indicador global y bastante razonable para utilizarlo como comparación de peso relativo entre naciones; que no quiere decir, obviamente, que países con PIB similares estén en una misma situación de poder.

Fonseca (2002) comenta que los programas de investigación en progreso enfocan el problema de los determinantes de la felicidad por diversos ángulos, entre estos se destacan: el estudio sobre lo que permite ciertos estados de conciencia más o menos agradables, la identificación de los factores personales, socioeconómicos y 
culturales, asociados a variaciones de bienestar subjetivo; las bases químicas, hormonales y neurobiológicas de las experiencias mentales y emocionales que llevan a alguien a sentirse más o menos feliz con la vida que tiene.

El objetivo de este artículo pretende colocar en debate la importancia de reflexionar sobre los indicadores objetivos y subjetivos de bienestar y revelar la importancia de pensar y considerar la utilización de indicadores socio-ambientales, que permiten incorporar una racionalidad socio-ambiental para lograr un desarrollo territorial sustentable a escala humana. Se pretende considerar el Índice de Desarrollo Humano (IDH) y el Ecological Footprint (Huella ecológica, según su traducción al español), colocando de esta manera, a estos indicadores como herramientas para establecer estrategias y toma de decisiones que permitan una planificación para el desarrollo territorial sustentable, considerando a las personas como sujetos y no como objetos.

Por medio de la presente reflexión sobre indicadores de sustentabilidad (socioambientales), y el cómo considerarlos en la elaboración de políticas, podría volver a las personas de una determinada comunidad, ciudad o país, actores más empoderados con énfasis en la sustentabilidad.

\section{Fundamentación teórica}

\section{¿Estamos como estamos porque somos como somos?}

El siglo XXI nos coloca nuevamente en el desafío de re-pensar y reflexionar sobre el actual modelo de desarrollo, notablemente globalizado, o más bien "bobalizado", donde el capitalismo, la competencia, desigualdad y la acumulación de riqueza parecen ser los únicos caminos a seguir. Este trabajo pone en tela de juicio que exista una democracia económica.

En este siglo XXI gran cantidad de la población vive en metrópolis, y en el mejor de los casos, en ciudades pequeñas. Las ciudades crecen año a año, convirtiendo al ser humano en un ser primordialmente urbano. Es un hecho que el Homo Sapiens que sabía convivir en armonía con la naturaleza, se convirtió en un "bomo urbanistus economistus” que nunca más se relaciona con ella, a no ser para apropiársela.

Según comenta Días (2002), los seres humanos ahora constituyen una especie predominantemente urbana, ya que el 70\% de la población de los Estados Unidos, Canadá, Europa Occidental y Japón son urbanas, y el 74\% de los latinoamericanos viven en ciudades. En Brasil, por ejemplo, aproximadamente el 80\% de la población vive en ciudades que no fueron diseñadas para soportar condiciones climáticas extremas o cambios drásticos en la temperatura (BORN, 2009). 
En este contexto, no cabe duda de que lo que sucede en el mundo urbano termina generando patologías socio-ambientales, socio-políticas, socio-culturales, socioeconómicas, que afectan a toda la biosfera. Por ejemplo, las ciudades son ahora responsables de la emisión de $3 / 4$ partes del dióxido de carbono mundial (DÍAS, 2002).

En el plano político, las patologías están marcadas por la ineficacia de las instituciones políticas frente a las elites y aquellos que controlan el poder. En el plano cultural, la fragmentación de las identidades socio-culturales y la pérdida de las formas tradicionales de vida son elocuentes. En el plano económico, la racionalidad instrumental, economicista - utilitarista, es señalada como la gran responsable de la enorme desigualdad, donde $1 / 4$ de la población concentra $3 / 4$ partes del PIB mundial. Y por último, en el plano ambiental los indicadores ambientales como la huella ecológica, o el informe del Intergovernmental Panel of Climate Change (IPCC), la World Meteorological Organization (WMO), y el United Nations Environmental Programme (UNEP), han advertido sobre los riesgos de continuar un modelo de desarrollo que no toma en cuenta los costos socioambientales derivados de las actividades productivas (SACHS, 1986; MAX-NEEF, 2001; PNUD, 2007; SAMPAIO, 2009; HENRÍQUEZ; ZECHNER; SAMPAIO, 2009).

Según Días (2002, p. 21)

quien nace en ciudades no se da cuenta, en su mayoría, de lo que sucede. Las luces brillantes, el ritmo frenético, las horas de trabajo, las horas de diversión, eliminan la posibilidad de dedicar un tiempo para reflexionar o percibir lo que está sucediendo, por otra parte, el agua disponible en los grifos, los alimentos disponibles en los supermercados (no para todos), les confiere una sensación poderosa de independencia, debidamente reforzada por los medios de comunicación, el apoyo a los procesos educativos, y por ejemplo, los recién casados no imaginan que para producir sus anillos de oro, fueron generadas dos toneladas de residuos vertidos en el medioambiente.

Van Bellen (2006, p. 13) llama la atención cuando dice

que en el lado exponencial del desarrollo tecnológico, que aumenta la expectativa de vida de los seres humanos y al mismo tiempo su capacidad de autodestrucción, ocurre un crecimiento significativo de utilización de materia y energía para responder a las necesidades de la sociedad. Esa demanda por bienes y servicios ocurre en toda la superficie del globo, pero su aprovechamiento no es uniforme. 
Es importante resaltar el texto de Silva (2009), cuando dice que el caso brasileño, con un $17 \%$ de bosque consumido, se encuentra en una situación límite. El gobierno brasileño necesita escoger entre liderar una transición para nuevos parámetros de desarrollo global o continuar siendo fiel al pasado, asegurando la sobrevivencia de un diseño basado en la sobreexplotación de los activos socioambientales para usufructo de pocos.

A su vez Sachs (2009) señala que Brasil reúne todas las condiciones para convertirse en un laboratorio, a escala mundial, de transición hacia las energías renovables ${ }^{1}$. Es oportuno aprovechar su capacidad para avanzar en el sentido de una biocivilización moderna, mostrando una posible salida para la humanidad.

\section{Pensando en una racionalidad socio-ambiental}

El bienestar subjetivo entendido como felicidad de vida, proporciona un criterio de validación empírica, siendo posible corroborar que los indicadores objetivos también se aproximan al bienestar. En otras palabras, es posible convalidar o refutar la práctica común de utilizar indicadores objetivos de ingreso y riqueza como indicadores de bienestar. Pero, la ciencia sabe que la hipótesis sobre bienestar, indicadores de ingreso y riqueza nunca han sido comprobados empíricamente (ROJAS, 2008).

Para Fonseca (2002) el bienestar humano abarca dos componentes básicos, una dimensión objetiva, considerada como aquella fácil de observar, asociada a indicadores numéricos. Y una dimensión subjetiva que se refiere a la experiencia interna del individuo, siendo que la confluencia de ambas dimensiones que permite encontrar la felicidad. La relación entre indicadores objetivos y subjetivos de bienestar, están lejos de obedecer a un patrón de comportamiento.

Son muchas las dificultades encontradas a la hora de discutir sobre el bienestar subjetivo. Reis (2008) afirma que, sumándose a la dificultad de medición de los indicadores subjetivos, es difícil obtener datos confiables y reales, porque muchas veces el problema real que aparece, es que el ser humano no puede transmitir lo que pasa en su interior. Para Fonseca (2002, p. 71) "las reales dificultades son de orden cognitivo y de comunicación".

En la actualidad los indicadores más utilizados son de carácter económico (objetivos) porque presentan una relativa facilidad al momento de la medición, en virtud de ser elaborados en base a datos cuantitativos. Los indicadores sociales y

\footnotetext{
${ }^{1}$ El mismo autor afirma que los biocombustibles pueden ser compatibles con el postulado de la seguridad alimentaria si se producen a partir de residuos agrícolas y forestales (SACHS, 2009).
} 
ambientales, y de bienestar, son más bien complejos, y presentan mayores dificultades para su medición.

No siempre el crecimiento económico puede ser traducido o comparado con los niveles de bienestar y felicidad, por parte de una población de una determinada sociedad. En este punto cabe destacar algunos ejemplos, décadas de fuerte crecimiento económico en Europa y Japón, en la mitad del siglo XX, muy poco o nada contribuían en modificar la proporción de individuos felices e infelices en la población de las respectivas naciones (FONSECA, 2002). Max-Neef (2007) llama la atención cuando dice que, a pesar que entre los años 1950 y 2000 el poder adquisitivo de los norte americanos se ha más que duplicado, pero el número de personas que se autodefinen como felices se ha mantenido prácticamente sin cambios.

Cuando nos fijamos en el indicador, es decir en el PIB (Producto Interno Bruto), podemos decir que es posible observar en la literatura, que los Estados Unidos genera como potencia económica casi el 30\% del PIB mundial (DUPAS, 2005), sin embargo, ese mismo país es responsable del 70\% de la basura mundial, y una huella ecológica que necesita de casi tres planetas tierra (DIAS, 2002).

Indicadores para un Desarrollo a Escala Humana: el pensamiento transdisciplinario de MaxNeef

Un trabajo de Max-Neef (2001) propone una perspectiva para pensar en otro desarrollo, o como el autor le llama, desarrollo a escala humana. Max-Neef entiende que los pilares fundamentales de desarrollo a escala humana son las satisfacciones de las necesidades humanas fundamentales, la generación de niveles crecientes de auto-dependencia y las articulaciones orgánicas de los seres humanos con la naturaleza, todas estas mediadas por la tecnología. A continuación se presenta la figura (1) que sintetiza lo que aquí hemos colocado. 
Figura 1 Pilares de Desarrollo a Escala Humana

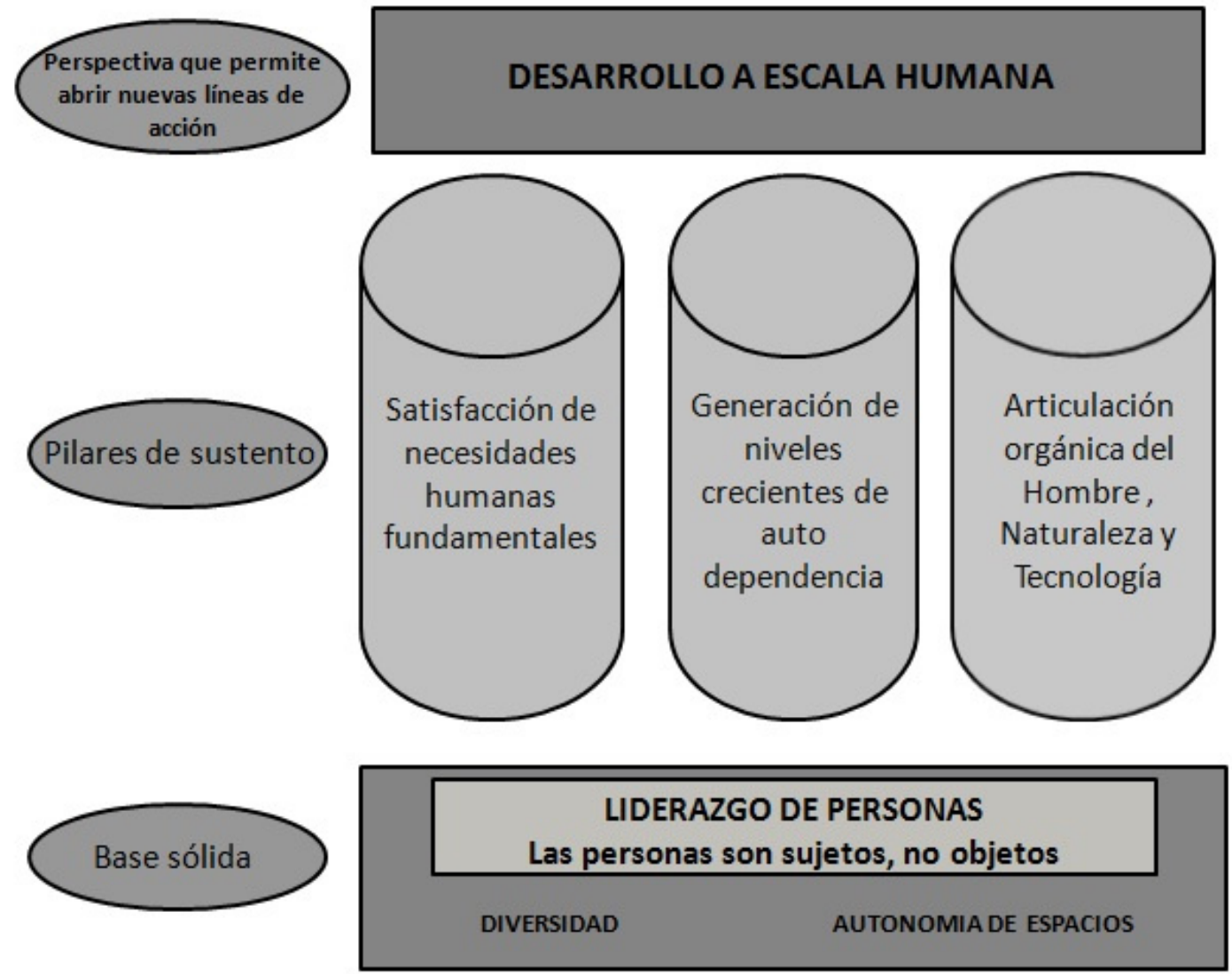

Fuente: Adaptado de Max-Neef (2001).

Como muestra la figura (1), estos pilares de desarrollo se construyen sobre una base sólida del protagonismo real de las personas, como sujetos en libertad. MaxNeef (2001) señala que la necesidad de mejorar la calificación de los procesos democráticos para el desarrollo a escala humana es posible, plausible y factible.

En el ámbito de las necesidades humanas fundamentales Max-Neef (2001) sostiene que es primordial reinterpretar los conceptos de necesidades y satisfactores, como también de los conceptos de pobreza y exclusión. Para Assis (2006), el bienestar es alcanzado con la atención de las necesidades que no se limitan al poder de adquisitivo del consumidor. Necesidades que deben ser consideradas a partir de un diagnóstico participativo para pensar en desarrollo. Tanto la riqueza, la pobreza y la inclusión o exclusión no necesariamente se refieren a la dimensión económica, sino más bien a la dimensión moral y emocional.

Bajo la perspectiva económica neoclásica, el desarrollo implica el aumento de la producción, asociada con la industrialización, y la acumulación de capital productivo. Esto puede ser medido a partir de indicadores de nivel de ingreso per cápita, la tasa de ocupación (desempleo), la distribución interna del ingreso, la 
existencia de infraestructura básica, entre otros. Todos estos indicadores son, en efecto, representativos de las condiciones para la atención de algunas necesidades que definen el bienestar. Sin embargo, se refieren solamente a una parte de las necesidades, definiendo solamente una parte del bienestar, es decir, miden solamente la dimensión material de las necesidades (ASSIS, 2006).

En este sentido Max-Neef (2001) coloca en discusión los conceptos de necesidad y satisfactor cuando plantea reinterpretarlos, afirmando que existe un error conceptual que no separa necesidad de satisfactor. Para nuestro autor, las necesidades humanas fundamentales, a diferencia de la propuesta piramidal de Maslow (1943), son pocas, finitas y clasificables, no son variables en el tiempo y en las diferentes culturas, siendo que lo que realmente cambia son los satisfactores. Las necesidades las clasifica en variables ontológicas (ser, tener, estar y hacer) y en variables axiológicas (subsistencia, protección, afecto, entendimiento, participación, ocio, creación, identidad y libertad). Esta reinterpretación de los conceptos de necesidades y satisfactores permite según el autor la construcción de una matriz de necesidades que se muestra en el cuadro 1.

\section{Cuadro 1 Matriz de necesidades humanas fundamentales}

(continua)

\begin{tabular}{|c|l|l|l|l|}
\hline \multicolumn{1}{|c|}{ Ser } & \multicolumn{1}{c|}{ Tener } & \multicolumn{1}{c|}{ Hacer } & \multicolumn{1}{c|}{ Estar } \\
\hline Subsistencia & $\begin{array}{l}\text { 1/ Salud física y } \\
\text { mental, } \\
\text { equilibrio, } \\
\text { solidaridad, } \\
\text { humor, } \\
\text { adaptabilidad }\end{array}$ & $\begin{array}{l}\text { 2/ Alimentación, } \\
\text { abrigo, trabajo }\end{array}$ & $\begin{array}{l}\text { 3/ Alimentar, } \\
\text { procrear, } \\
\text { descansar, } \\
\text { trabajar }\end{array}$ & $\begin{array}{l}\text { 4/ Entorno vital, } \\
\text { entorno social }\end{array}$ \\
\hline Protección & $\begin{array}{l}\text { 5/ Cuidado, } \\
\text { adaptabilidad, } \\
\text { autonomía, } \\
\text { equilibrio, } \\
\text { solidaridad }\end{array}$ & $\begin{array}{l}\text { 6/ Sistema de } \\
\text { seguros, de salud, } \\
\text { ahorro, seguridad } \\
\text { social, } \\
\text { legislaciones, } \\
\text { derecho, familia, } \\
\text { trabajo }\end{array}$ & $\begin{array}{l}\text { 7/ Cooperar, } \\
\text { prevenir, } \\
\text { planificar, } \\
\text { cuidar, curar, } \\
\text { defender }\end{array}$ & $\begin{array}{l}\text { 8/ Entorno vital, } \\
\text { entorno social, } \\
\text { morada }\end{array}$ \\
\hline Afecto & $\begin{array}{l}\text { 9/ Autoestima, } \\
\text { solidaridad, } \\
\text { respeto, } \\
\text { tolerancia, } \\
\text { generosidad, } \\
\text { receptividad, } \\
\text { pasión, voluntad, } \\
\text { sensualidad, } \\
\text { humor }\end{array}$ & $\begin{array}{l}\text { 10/ Amistades, } \\
\text { parejas, familia, } \\
\text { animales } \\
\text { domésticos, } \\
\text { plantas, jardines }\end{array}$ & $\begin{array}{l}\text { amor, acariciar, } \\
\text { expresar, } \\
\text { emociones, } \\
\text { compartir, } \\
\text { cuidar, cultivar, } \\
\text { apreciar }\end{array}$ & $\begin{array}{l}\text { 11/ Hacer el } \\
\text { intimidad, hogar, } \\
\text { espacios de } \\
\text { encuentro }\end{array}$ \\
\hline
\end{tabular}


(continuação)

\begin{tabular}{|c|c|c|c|c|}
\hline Entendimiento & $\begin{array}{l}\text { 13/ Conciencia } \\
\text { crítica, } \\
\text { receptividad, } \\
\text { curiosidad, } \\
\text { asombro, } \\
\text { disciplina, } \\
\text { intuición, } \\
\text { racionalidad }\end{array}$ & $\begin{array}{l}\text { 14/ Literatura, } \\
\text { maestros, } \\
\text { métodos, políticas } \\
\text { educacionales, } \\
\text { políticas } \\
\text { comunicacionales }\end{array}$ & $\begin{array}{l}\text { 15/ Investigar, } \\
\text { estudiar, } \\
\text { experimentar, } \\
\text { educar, analizar, } \\
\text { meditar, } \\
\text { interpretar }\end{array}$ & $\begin{array}{l}\text { 16/ Ámbitos de } \\
\text { interacción } \\
\text { formativa: } \\
\text { escuelas, } \\
\text { universidades, } \\
\text { academias, } \\
\text { agrupaciones, } \\
\text { comunidades, } \\
\text { familia }\end{array}$ \\
\hline Participación & $\begin{array}{l}17 / \\
\text { Adaptabilidad, } \\
\text { receptividad, } \\
\text { solidaridad, } \\
\text { disposición, } \\
\text { convicción, } \\
\text { entrega, respeto, } \\
\text { pasión, humor }\end{array}$ & $\begin{array}{l}18 / \text { Derechos, } \\
\text { responsabilidades, } \\
\text { obligaciones, } \\
\text { atribuciones, } \\
\text { trabajo }\end{array}$ & $\begin{array}{l}\text { 19/ Afiliarse, } \\
\text { cooperar, } \\
\text { proponer, } \\
\text { compartir, } \\
\text { discrepar, acatar, } \\
\text { dialogar, } \\
\text { acordar, opinar }\end{array}$ & $\begin{array}{l}\text { 20/ Ámbitos de } \\
\text { interacción } \\
\text { participativa: } \\
\text { partidos, } \\
\text { asociaciones, } \\
\text { iglesias, } \\
\text { comunidades, } \\
\text { vecindarios, } \\
\text { familias }\end{array}$ \\
\hline Ocio & $\begin{array}{l}\text { 21/ Curiosidad, } \\
\text { receptividad, } \\
\text { imaginación, } \\
\text { despreocupación, } \\
\text { humor, } \\
\text { tranquilidad, } \\
\text { sensualidad }\end{array}$ & $\begin{array}{l}\text { 22/ Juegos, } \\
\text { espectáculos, } \\
\text { fiestas, calma }\end{array}$ & $\begin{array}{l}\text { 23/ Divagar, } \\
\text { abstraerse, } \\
\text { soñar, añorar, } \\
\text { fantasear, } \\
\text { evocar, relajarse, } \\
\text { divertirse, jugar }\end{array}$ & $\begin{array}{l}\text { 24/ Privacidad, } \\
\text { intimidad, } \\
\text { espacios de } \\
\text { encuentro, } \\
\text { tiempo libre, } \\
\text { ambientes, } \\
\text { paisajes }\end{array}$ \\
\hline Creación & $\begin{array}{l}\text { 25/ Pasión, } \\
\text { voluntad, } \\
\text { intuición, } \\
\text { imaginación, } \\
\text { audacia, } \\
\text { racionalidad, } \\
\text { autonomía, } \\
\text { inventiva, } \\
\text { curiosidad }\end{array}$ & $\begin{array}{l}\text { 26/ Habilidades, } \\
\text { destrezas, } \\
\text { método, trabajo }\end{array}$ & $\begin{array}{l}\text { 27/ Trabajar, } \\
\text { inventar, } \\
\text { construir, idear, } \\
\text { componer, } \\
\text { diseñar, } \\
\text { interpretar }\end{array}$ & $\begin{array}{l}\text { 28/ Ámbitos de } \\
\text { producción y } \\
\text { retroalimentació } \\
\text { n: talleres, } \\
\text { ateneos, } \\
\text { agrupaciones, } \\
\text { audiencias, } \\
\text { espacios de } \\
\text { expresión, } \\
\text { libertad temporal }\end{array}$ \\
\hline Identidad & $\begin{array}{l}\text { 29/ Pertenencia, } \\
\text { coherencia, } \\
\text { diferenciación, } \\
\text { autoestima, } \\
\text { asertividad }\end{array}$ & $\begin{array}{l}\text { 30/ Símbolos, } \\
\text { lenguaje, hábitos, } \\
\text { costumbres, } \\
\text { grupos de } \\
\text { referencia, } \\
\text { sexualidad, } \\
\text { valores, normas, } \\
\text { roles, memoria } \\
\text { histórica, trabajo }\end{array}$ & $\begin{array}{l}\text { 31/ } \\
\text { Comprometerse, } \\
\text { integrarse, } \\
\text { confrontarse, } \\
\text { definirse, } \\
\text { conocerse, } \\
\text { reconocerse, } \\
\text { actualizarse, } \\
\text { crecer }\end{array}$ & $\begin{array}{lr}32 / & \text { Socio- } \\
\text { ritmos, entornos } \\
\text { de } \\
\text { cotidianeidad, } \\
\text { ámbitos de } \\
\text { pertenencia, } \\
\text { etapas } \\
\text { madurativas }\end{array}$ \\
\hline
\end{tabular}


(conclusão)

\begin{tabular}{|c|l|l|l|l|}
\hline Libertad & $\begin{array}{l}\text { 33/ Autonomía, } \\
\text { autoestima, } \\
\text { voluntad, pasión, } \\
\text { asertividad, } \\
\text { apertura, } \\
\text { determinación, } \\
\text { audacia, rebeldía, } \\
\text { tolerancia }\end{array}$ & $\begin{array}{l}35 / \text { Discrepar, } \\
\text { derechos } \\
\text { optar, } \\
\text { diferenciarse, } \\
\text { arriesgar, } \\
\text { conocerse, } \\
\text { asumirse, } \\
\text { desobedecer, } \\
\text { meditar }\end{array}$ & $\begin{array}{l}\text { 36/ Plasticidad } \\
\text { espacio-temporal }\end{array}$ \\
\hline
\end{tabular}

Fuente: Max-Neef (2001).

Pensar en las necesidades como infinitas, es un error conceptual. Hay que diferenciar necesidad de "satisfacción". Los satisfactores son la forma o el medio por los cuales se puede satisfacer las necesidades humanas fundamentales, y son estos los que cambian con el tiempo y diferentes culturas. En este sentido, el consumo claramente no es una necesidad, si no una satisfacción.

\section{Entendiendo los indicadores}

El objetivo principal de este estudio es abordar la importancia de la incorporación de una racionalidad social y ambiental en el debate sobre los indicadores, que permita repensar el desarrollo en una escala humana. El termino indicador tiene origen en el verbo del latín "Indicare", que quiere decir "revelar" (GARCÍA; GUERRERO, 2006). La aplicación del concepto indicador de sustentabilidad, revela el parámetro que entrega la información sobre el estado de la relación entre sociedad y naturaleza.

Los indicadores pueden ser definidos como variables dotadas de significados, derivados de su propia configuración científica, que reflejan de forma sintética un interés social por el ambiente, posibilitando su inclusión coherente en el proceso de toma de decisiones (HENRÍQUEZ, 2009).

Los indicadores para la sustentabilidad son herramientas capaces de evaluar progresos hacia el desarrollo sustentable. El proceso de elaboración de los indicadores socio-ambientales es lento y complejo, y requiere de enfoques específicos para el trabajo, donde los investigadores trabajen con visión global, pero actuando localmente. Por lo tanto, es imprescindible que exista interacción e integración de las diferentes áreas de conocimiento (científico y no-científico) de diferentes disciplinas.

Según Besserman (2003), hubo una escasez de información local, regional y mundial. La producción de estadísticas e indicadores sobre el desarrollo sostenible es insuficiente y precaria, las carencias superan con creces la oferta de 
información. Esto se debe a la aparición misma de los problemas ambientales, tanto en relación con el despertar de la conciencia ecológica a escala mundial, como la evolución acelerada de los daños al medio ambiente en las últimas décadas y su propagación.

Relevancia de los indicadores socio-ambientales: IDH y Huella Ecológica, justificando la racionalidad socio-ambiental

En una racionalidad predominantemente económica, la idea de desarrollo aparece como el aumento de la producción y el consumo. El proceso productivo se alimenta de la producción, y por otra parte, responde a las necesidades de los consumidores. Desde esta perspectiva, las necesidades deben siempre mantenerse y ampliarse, mientras mayor sea el consumo mayor será la producción. De esta manera el funcionamiento del sistema continúa generando puestos de empleos y beneficios.

En esa racionalidad, el bienestar social e individual es medido objetivamente por el aumento del volumen de consumo, de la producción y de la generación de empleos. Las técnicas utilizadas para medir las variables subjetivas, como es el caso de las necesidades y preferencias de los consumidores, son limitadas, incompletas o reducidas, de esta forma los análisis que apoyan estas medidas para el bienestar social e individual, también son incompletos (ASSIS, 2006).

Una de las características más llamativas del actual modelo de desarrollo hegemónico-económico, es no tomar en cuenta los costos socio-ambientales resultantes de las actividades de producción industrial. Por ese motivo, se hace necesaria la incorporación de aquellos costos, para influir en los procesos de toma de decisiones y en la elaboración de políticas públicas. Por ejemplo, una de las maneras de calcular estos costos es por medio de los indicadores socioambientales, que forman parte de un movimiento que gana fuerza y que, aunque rara vez se utilizan, tienen gran importancia.

Los indicadores son necesarios para monitorear el progreso en distintas dimensiones, funcionando como herramientas de apoyo para quienes toman las decisiones, o para los responsables de la elaboración de políticas en todas las áreas, permitiendo mantener el enfoque hacia el desarrollo sustentable (GARCÍA; GUERRERO, 2006). Además, los indicadores pueden servir de alerta para prevenir amenazas como los impactos económicos, sociales y ambientales de una actividad en particular. También pueden ser útiles como herramientas para difundir ideas, pensamientos y valores. El objetivo de los indicadores socioambientales para un desarrollo más sustentable, es promover una mayor conciencia acerca de las implicancias de la problemática ambiental y el desarrollo. 
El Índice de Desarrollo Humano (IDH) fue creado por el economista paquistaní Mahbub ul Haq, con la colaboración del Premio Nobel de Economía de 1998, el indio Amartya Sen, para medir el nivel de desarrollo humano de los países a partir de indicadores de educación (alfabetización), longevidad (esperanza de vida al nacer) e ingresos (PIB per cápita). Las variaciones del IDH, como el IDH-M (Índice de Desenvolvimiento Humano Municipal), miden los mismos fenómenos, pero son adaptados para evaluar las condiciones de núcleos sociales menores.

En 1995, el Programa de las Naciones Unidas para el Desarrollo (PNUD), difundió por medio de un informe sobre el IDH, los componentes esenciales del paradigma de desarrollo humano (FERREIRA, 1998):

a) Productividad: Posibilidad de que las personas aumenten su productividad y participen plenamente en el proceso de generación de insumos.

b) Equidad: Necesidad de las personas de tener acceso e igualdad de oportunidades, eliminando las barreras que obstruyen las oportunidades económicas y políticas.

c) Sustentabilidad: Garantizar el acceso no solamente a las generaciones actuales, sino también futuras. Basándose en todas las formas de capital: humano, físico y ambiental.

d) Potenciación: Efectuado por las personas y no sólo para ellas. Participación efectiva de las decisiones y procesos.

Aunque el concepto de Desarrollo Humano definido por el PNUD es bastante utilizado hoy en día, esta cuestión, a pesar de ser utilizada recientemente, no es nueva, existiendo concepciones anteriores. Es importante señalar que el termino desarrollo es generalmente utilizado, explícita o implícitamente, en relación a otro, subdesarrollo. El desarrollo estaría asociado a la prosperidad y el subdesarrollo a la carencia. Conforme a esta perspectiva, son ubicados como polos opuestos de: industrialización y producción en masa versus la estructura productiva artesanal y de subsistencia; moderno versus tradicional; países colonizadores versus países colonizados; centro versus periferia; términos de comercio dominantes versus dependientes; países deudores versus acreedores; entre otros.

Como no es de extrañar, generalmente entre los países con mayor IDH están los países desarrollados, notándose una gran disparidad entre los países del hemisferio sur y del norte, o sea, centro-periferia. La esencia del desarrollo humano se centra en tres bases fundamentales que buscan explicar y entender el proceso de desarrollo como concepto más amplio y complejo, es decir, el desarrollo para las personas.

La primera base se relaciona con el hecho de que el actual modelo de desarrollo, de carácter meramente economicista, relega al ser humano en segundo plano, siendo crucial el establecimiento de una nueva forma de compartir los frutos del crecimiento económico por la población, esto es, el aumento del volumen de los 
recursos destinados a la expansión y mejoría de los servicios públicos, sobre todo salud y educación. La segunda se relaciona con los recursos que cada país debería invertir para proporcionar un desarrollo humano, esto es, la creación de un ambiente propicio para que los humanos puedan explorar sus potencialidades y que estas se vuelvan mejores durante el proceso.

Arruda (2000), en ese sentido, enfatiza en lo que denomina "auto-desarrollo": Un desarrollo generado o impulsado desde de abajo para arriba y de dentro para fuera, cuya característica esencial es la de estar mucho más centrado en el ser humano y lo social, sugiriendo la participación del individuo (dimensión personal), de la sociedad civil, de las empresas y unidades políticas (dimensión de comunidad) como factor clave para definir la dirección del desarrollo local.

\section{Una lectura rápida sobre el informe del Planeta Vivo (2006 y 2008)}

El Informe del Planeta Vivo o Living Planet Report, es una red de esfuerzos de equipos multidisciplinarios que trabajan para describir el estado actual de la biodiversidad en niveles globales, para medir la presión que los seres humanos ejercemos sobre los ecosistemas. El informe fue creado en 1998 por WWF (World Wildlife Fund), y desde entonces sus previsiones son alarmantes sobre el estado de nuestro planeta (LIVING PLANET REPOR'T, 2006).

El Informe del Planeta Vivo es calculado en base a dos índices, el Índice de Planeta Vivo y la Huella Ecológica. El primero señala el estado actual de los ecosistemas del planeta, y es medido por el estudio o monitoreo de 1.686 especies emblemáticas de vertebrados en todas las regiones del mundo. El segundo índice muestra el alcance y el tipo de demanda que la humanidad está imponiendo en dichos ecosistemas estudiados por el Informe del Planeta Viviente (WWF, 2008).

Según el Informe del Planeta Vivo (2006), las noticias no son nada alentadoras y confirman que estamos como estamos, por seguir consumiendo los recursos naturales, es decir, la biodiversidad, en un ritmo superior a su capacidad de renovación -resiliencia. Este mismo informe nos muestra una Huella Ecológica que se ha más que triplicado desde 1961 al 2006.

La reciente depresión financiera de la economía mundial es una muestra de lo que sucede cuando gastamos más de lo que tenemos. Sin embargo, esto ni se compara con la preeminencia de los problemas más graves del Calentamiento Global ocasionados por el sobre-giro ecológico. De la misma manera que el gasto desmedido genera una recesión mundial, el consumo o su panacea, "el consumismo", está acabando con el capital natural del planeta (WWF, 2008).

Nuestra Huella Ecológica global excede actualmente en 30\% la capacidad de carga del planeta para regenerarse. En el Informe del Planeta Vivo de 2006, ya se 
señalaba que para el año 2050 necesitaríamos de dos planetas tierra, el informe de 2008, más pesimista, anticipa este escenario para mediados de la década del 2030. (WWF, 2006; 2008).

El gran desafío de estas proyecciones, es que no basta conocer los problemas, en realidad necesitamos comprender que tenemos solamente un planeta y que negligentemente pareciera que su capacidad de carga es grande, e ilimitada (WWF, 2008).

Vamos a observar dos gráficos a continuación, Gráfico 1 Índice del Planeta Vivo y Gráfico 2 Huella Ecológica de la Humanidad que ilustra las condiciones en que nos encontramos actualmente.

\section{Gráfico 1 Índice del Planeta Vivo, 1970-2005}

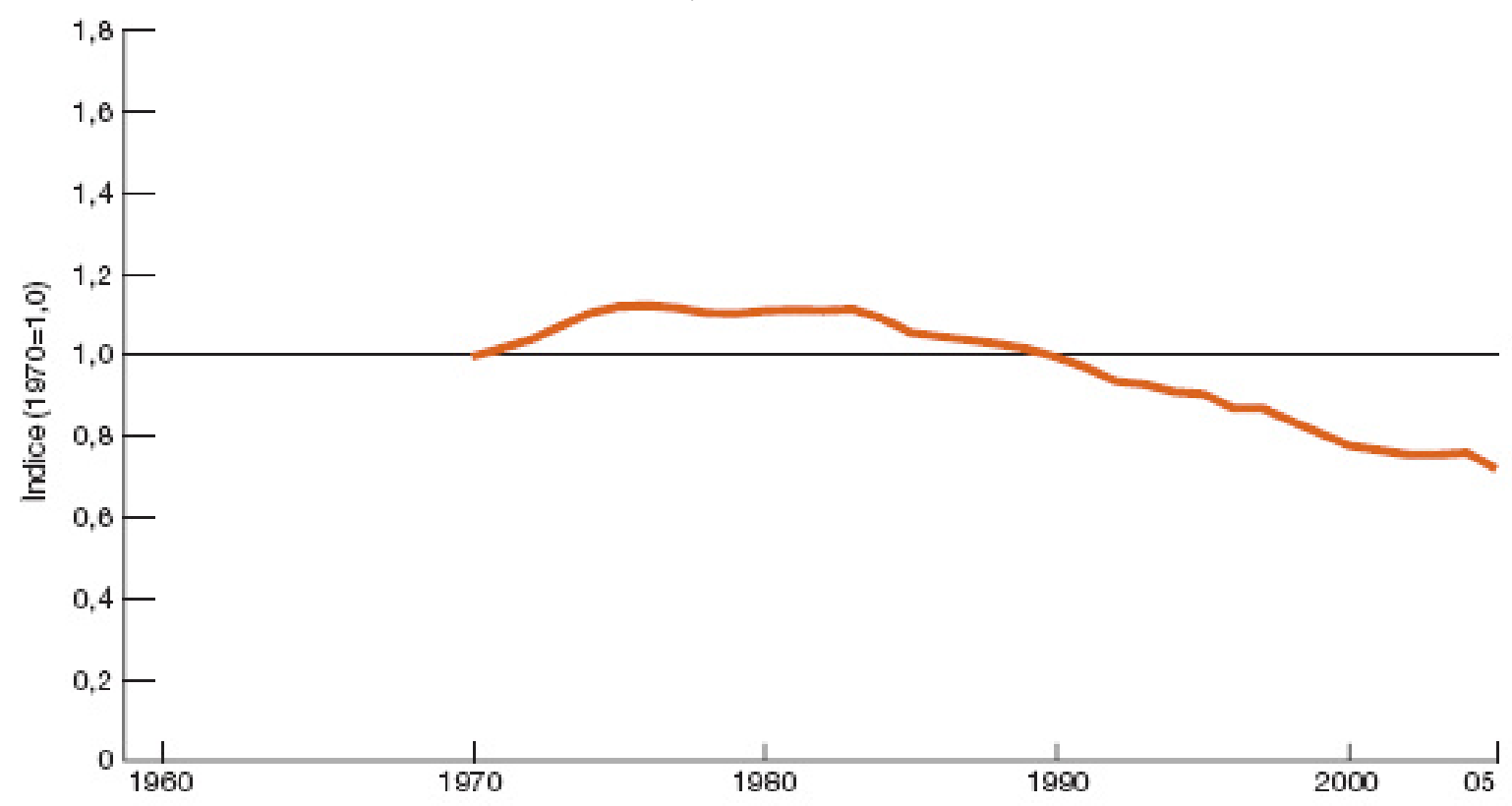

Fuente: Informe del Planeta Vivo (2008). 


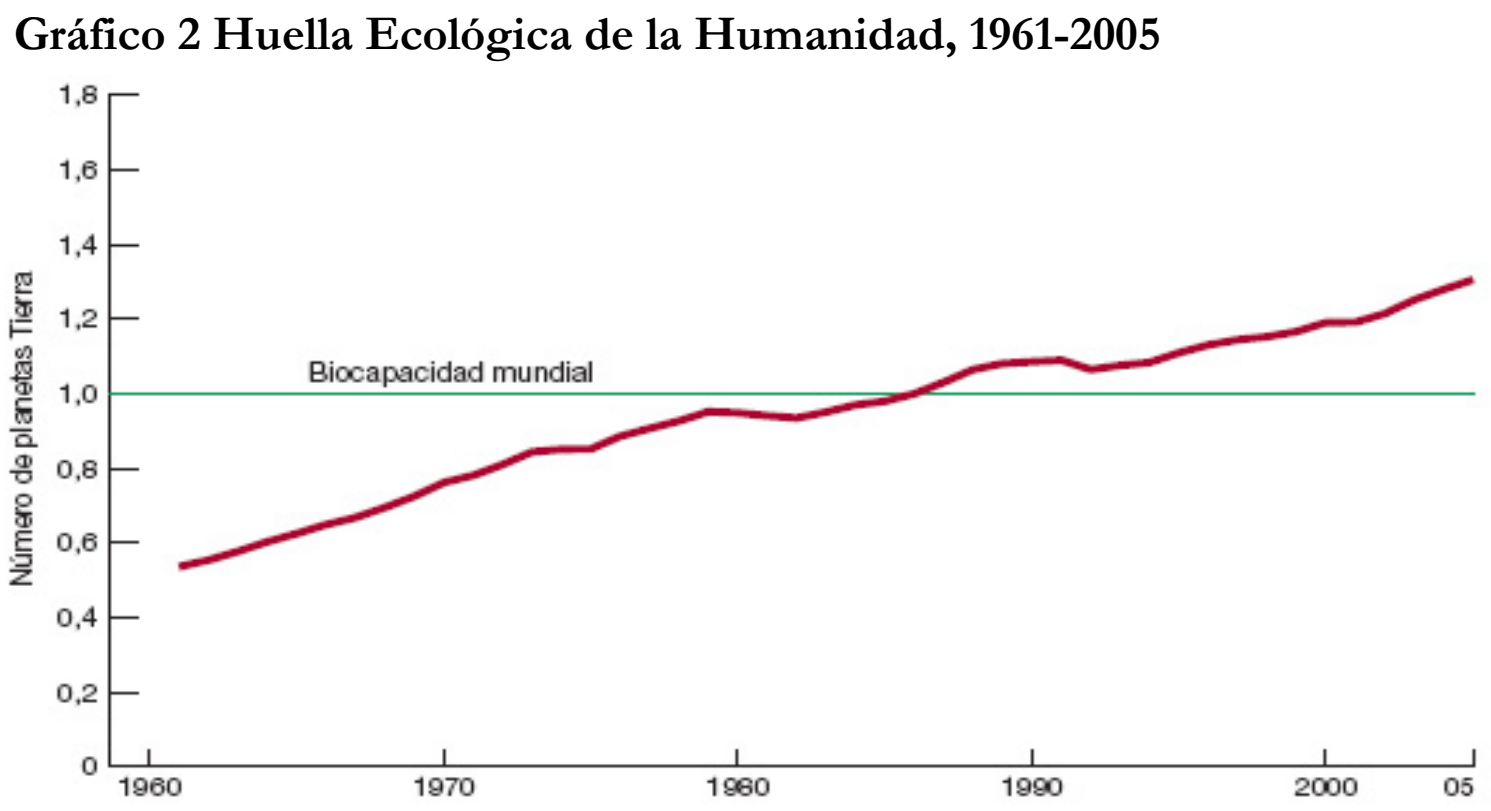

Fuente: Informe del Planeta Vivo (2008).

$\mathrm{Al}$ analizar los gráficos 1 y 2 , se percibe que la demanda de la humanidad sobre el planeta se ha más que duplicado en los últimos 45 años, resultado del crecimiento poblacional, y sobre todo, de su actitud consumista. Es necesario también destacar, que en la década del 60 , casi todos los países del mundo tenían una capacidad más que suficiente para satisfacer sus propias demandas ecológicas. Ya en el año 2005, los países industrializados presentan una deuda ecológica - Huella Ecológica alta - por lo que ya deberían importar recursos de países con excedente de capacidad ecológica - Huella Ecológica baja (WWF, 2008).

El Informe del Planeta Vivo (2008), reporta que más de tres cuartas partes de la población mundial viven en naciones que poseen deuda ecológica, proveniente de un estilo de vida oriundo del crecimiento económico, lo que quiere decir que estarían necesariamente extrayendo capital natural de otras partes del mundo. La pregunta que se puede hacer es: ¿De qué parte del mundo?

\section{Consideraciones finales}

Para concluir el presente estudio es necesario preguntarse: ¿Cómo medir el desarrollo? Hace bastante tiempo los organismos internacionales, gobiernos, instituciones académicas e investigadores intentan responder esta pregunta. Por lo tanto, la construcción de indicadores ha sido la herramienta más utilizada por aquellos que objetivamente pretende medir el desarrollo de países, regiones, localidades y comunidades. Aunque a menudo sólo reciben las tasas de crecimiento a través de las variables económicas, configurando de esta forma los 
indicadores objetivos que se presuponen válidos para la elaboración de políticas públicas, aunque estos no hayan sido validados científicamente.

El bienestar subjetivo también se ha visto influenciado por las percepciones, por las condiciones objetivas de posición socio-económica y de satisfacción de las necesidades materiales. Las percepciones de las personas generalmente no guardan una estrecha relación con las condiciones objetivas del nivel de vida. Por lo que existen diferencias considerables entre lo que dicen los indicadores objetivos y los indicadores subjetivos de bienestar.

En este sentido, el PIB permaneció por un largo periodo siendo utilizado para medir la riqueza de las naciones. Acabando por ser blanco de críticas debido a su restringida cobertura y por no responder a las necesidades actuales, siendo implementado y usado el Îndice de Desarrollo Humano (IDH) como contrapunto, con amplia aceptación a nivel internacional por ser considerado como un indicador más completo - a pesar de sus limitaciones- al incorporar dimensiones socio-económicas.

En un proceso evolutivo, acompañado de las discusiones emergentes en torno a la sustentabilidad, se cuestiona actualmente la posibilidad de creación de indicadores más amplios, capaces de contemplar las variables económicas, sociales y ambientales, y al mismo tiempo, ser aceptados científicamente en lo que se refiere a credibilidad de los resultados. Tal preocupación gana fuerza en un contexto en que se cristaliza la problemática ambiental, tanto a nivel global, como nacional, regional y asimismo local.

Las contribuciones del Índice de Desarrollo Humano (IDH) y la Ecological Footprint (Huella Ecológica) se basan en el intento de considerar otros indicadores como herramientas para el establecimiento de estrategias y toma de decisiones que visen la planificación para el desarrollo a escala humana. La presente discusión pretende demostrar que la implementación de indicadores socio-ambientales pasa a ser una cuestión fundamental. En este sentido son necesarios otros estudios sobre indicadores socio-ambientales.

Junto a las discusiones emergentes en torno a la sustentabilidad, se cuestiona actualmente la posibilidad de creación de indicadores más amplios, capaces de contemplar variables económicas, sociales y ambientales, y al mismo tiempo, ser validados por los grupos de influencia. Sin embargo, con la creciente divulgación de la problemática socio-ambiental, gana fuerza la necesidad de pensar alternativas, a lo que socialmente llamamos desarrollo.

Dado el complejo escenario de hoy, caminar en la dirección de nuevos indicadores es una emergencia. Sin embargo, esto requiere de enfoques que abandonen la perspectiva unidisciplinaria y el raciocinio cartesiano. El ambiente es complejo, incierto e inestable, exige enfoques eco-socio-sistémicos integrados, transdisciplinarios, que reconozcan nuevos campos de investigación, capaces de indicar caminos alternativos para los desafíos que se presentan. 
Desde el desarrollo a escala humana se puede afirmar que las escenas locales dan cuenta de conflictos y tensiones entre comunidades locales y empresas que ven en los recursos naturales un medio de vida, en un caso, y uno de lucro, en el otro. En ambos casos el tema del desarrollo y la sustentabilidad abren los flancos de la naturaleza como recurso, por una parte, y de la sociedad, como continuidad de la existencia social, por otro. Los casos son ilustrativos de la forma como las prácticas socio-productivas que generan consecuencias socio-ambientales, determinan las características del paisaje, a la vez que levantan interrogantes acerca de las formas como se mide la sustentabilidad y acerca de cómo se puede promover la gestión participativa de territorios.

\section{Referências}

ARRUDA, M. Globalização e sociedade civil. Repensando o cooperativismo no contexto da cidadania ativa. In: ARRUDA, M.; BOFF, L. Globalização: desafios socioeconômicos, éticos e educativos. Uma visão a partir do Sul. Petrópolis: Editora Vozes, 2000, p. 49-102.

ASSIS, W. A. P. Estudo sobre desenvolvimento, bem-estar e necessidades humanas para uma econômica da complexidade. Tese (Doutorado em Meio Ambiente e Desenvolvimento) Universidade Federal do Paraná, Curitiba, 2006.

BESSERMAN, S. Indicadores: a lacuna das informações ambientais. In: TRIGUEIRO, A. (Coord.). Meio ambiente no século 21: 21 especialistas falam da questão ambiental nas suas áreas de conhecimento. Rio de Janeiro: Sextante, 2003, p. 90-105.

BORN, R. Mudanças climáticas e verdades inconvenientes. Le Monde Diplomatique Brasil, Julho, 2009.

DIAS, G. F. Pegada Ecológica e sustentabilidade humana: as dimensões humanas das alterações ambientais globais - um estudo de caso brasileiro (como metabolismo ecossistêmico urbano contribui para as alterações ambientais globais). São Paulo: Gaia, 2000.

DUPAS, G. Atores e poderes na nova ordem global. Assimetrias, instabilidades e imperativos de legitimação. São Paulo: Editora UNESP, 2005.

FERREIRA, C. Desenvolvimento humano revisitado. Brief Papers. Lisboa, 1998. 
FONSECA, E. G. Felicidade: diálogos sobre o bem estar na civilização. São Paulo: Companhia das letras, 2002.

GARCÍA, S.; GUERRERO M. Indicadores de sustentabilidad ambiental en la gestión de espacios verdes. Parque urbano Monte Calvario, Tandil, Argentina. Revista de Geografía Norte Grande, n. 35, p. 45-57, 2006.

HENRÍQUEZ, C. A construção participativa de indicadores territoriais socioambientais para o desenvolvimento regional sustentável. Análise propositiva para as comunidades do Rio Sagrado, Morretes (PR): Zona de Educação para o Ecodesenvolvimento. Universidade Regional de Blumenau. Tesis de Magister, 2009.

HENRÍQUEZ, C.; ZECHNER, T.; SAMPAIO, C. A. Turismo e suas interações nas transformações do espaço rural. In: SANTOS; DE SOUZA. Turismo no espaço rural. Editora Manole, 2009.

HOBSBAWM, E. A era dos extremos: o breve século XX-1914-1991. São Paulo: Schwarcz, 1996.

MASLOW, A. A theory of human motivation. Psychological Review, 50, p. 37096, 1943.

MAX-NEEF, M. Desarrollo a Escala Humana. Montevideo: Nordam comunidade, 2001.

Introducción a la Conservación Biológica, desde una perspectiva crítica y multidisciplinaria. Curso do Programa de Honor da Universidade Austral de Chile. A disciplina foi coordenada pelo Prof. Dr. Iñaki Ceverio, durante o primeiro semestre de 2007.

MORAN, T. Inversión extranjera directa y desarrollo. Tradução Sergio Gerardo López. Ciudad de México: Oxford University, 2000.

PNUD. Programa de las Naciones Unidas para el Desarrollo. Informe sobre Desarrollo Humano - 2005. Madrid: Ediciones Mundi-Prensa, 2007.

REIS, R. Reflexões e trabalho sobre indicadores objetivos e subjetivos de bem estar. Baseado em Fonseca E. G. 2002. Apresentado na disciplina Processos de Desenvolvimento do Mestrado em Desenvolvimento Regional, 2008.

ROJAS, M. E1 bienestar subjetivo en México y su relación con los indicadores objetivos. Consideraciones para la política pública, p. 21. 2005. 
SACHS, I. Ecodesenvolvimento: crescer sem destruir. São Paulo: Ed Vértice, 1986.

. O caminho para um outro paradigma. Le Monde Diplomatique Brasil, 2009.

SAMPAIO, C. A. Ecossocioeconomia das organizações. Blumenau: Edifurb, 2009.

SEN, A. Desenvolvimento como liberdade. Trad. Laura Teixeira Motta. São Paulo: Companhia das Letras, 2000.

SILVA, M. O arcaico e o novo na Amazonia. Le Monde Diplomatique Brasil, 2009.

VAN-BELLEN, H. M. Indicadores de sustentabilidade: uma análise comparativa. São Paulo: FGV, 2006.

WWF. World Wildlife Fund. Informe del Planeta Vivo. 2006. . World Wildlife Fund. Informe del Planeta Vivo. 2008.

* Este trabajo surge en el contexto del proyecto Fondecyt: F-1090465 "Paisajes de Agua y sustentabilidad en la cuenca del río Valdivia"

Endereço para correspondência:

Christian Henriquez.Zuñiga - christianhen@gmail.com

Casa 4A, Campus Isla Teja

5090000 Valdivia/Los Ríos, Chile

Guillermo Pacheco Habert - guillermopachecohabert@gmail.com

Casa 4A, Campus Isla Teja

5090000 Valdivia/Los Ríos, Chile

46 | Revista Brasileira de Desenvolvimento Regional, Blumenau, 2 (2), P. 27-46, primavera de 2014 